\title{
The Application of Color in Interior Design
}

\author{
Yingfeng Kuang \\ South China university of Technology \\ Guangzhou, China 510000
}

\author{
Weihai Zhang* \\ South China university of Technology \\ Guangzhou, China 510000 \\ *Corresponding Author
}

\begin{abstract}
Color is an important constituent in interior design. With the progress of social culture and the improvement of human aesthetic consciousness, many people begin to master the law of color, and it is well versed in the expression that color can bring to the space. Color express in the room in according with their rendering property, giving the impression of fresh and elegant, simple and generous, respected comfortable, natural and noble life. And it can result in the perfect combination of practical and artistic. It is an indispensable part of interior design. Color, which creates a very modern and personalized atmosphere of life, differs from light into shadow to create an extraordinary visual effect.
\end{abstract}

Keywords—color; color application; interior design

\section{INTRODUCTION}

Color is an expressive language; different colors can express different function. Bright color has a diffuse visual function which can make the object bigger than it really is; while color with low brightness can make the object smaller. Objects with different shapes, placement, and space combinations are all in association with the color arrangements. Good color application can not only be a good expression of the indoor atmosphere, so that the overall indoor space environment can be more harmonious, but it also can bring people pleasure and relaxation. Color does have an immeasurable role.

The well-known artist Rodin has said: "Beauty is harmony", so if the room has a very reasonable and effective use of color, then you can live in the indoor space and create a beautiful and fun living environment with a sense of richness.

\section{COLOR ANALYS}

\section{A. Hue Contrast}

A good indoor environment can promote a healthy development of human body and mind, and it can also promote brain's intellectual development. A professional survey found that the beautiful atmosphere can enhance brains' efficiency by $10 \%-40 \%$, the color in which a great impact. There are many designers refer color to "the nearest luxury". Because as long as the color are designed and arranged properly, even if the material within the house is not qualify enough, it can still have a good visual effect.
Hue refers to a type of color. And a hue contrast refers to the comparison of different colors. According to the order on the color wheel, we can divide hue contrast into different types. Colors near each other on the color wheel are called weak contrast while colors far from each other are called strong contrast, such as red and green. Such a contrast can have great visual impact and stimulation.

\section{B. Color Brightness Contrast}

The brightness of a color refers to its shade and depth. The brighter a color is, the lighter it makes us feel. By adding black or white to the same color can make it a different shading effect. A bright color feels beautiful and lively, while a less bright color feels more like a gray tone, giving the impression of warmth and simplicity.

Brightness contrast means putting two or more colors together, the brighter color will look even brighter; on the contrary, the less bright color looks much dimmer. The result of Color brightness contrast can be very effective in influencing the level and the size of space and objects.

\section{Color Purity}

Purity means how bright or dull a color is. Usually a high purity color will make the indoor environment looks bright, vivid and full of vitality. Low purity color makes the interior space introverted, old and stable. Usually a bright color's purity is not too high, generally it is relatively low, and the color feels close to gray while the middle tone has a middle purity. It is neither bright nor dull, which makes them an important choice for interior design.

The purity comparison is a visual difference due to the different saturation of colors. When compare a colorful green and a green with gray in it, you can clearly see which color is brighter and which is browner. This contrast is actually the contrast of the color purity.

\section{COLOR EMOTION}

\section{A. Visual Psychology}

Visual is the first way to receive information for us human beings. When seeing different colors, our mind will inevitably produce subtle changes. A professional physicist's survey shows that color can affect people's brain waves. When the brain waves encountered red, the first feeling will be nervous and excited, but when the brain waves encountered blue, we feel soothing. While other psychologists have found that color has a certain impact on 
people's minds. For instance, blue makes people feel a little cold, and red makes people feel warm, the reason why different color has different psychological feelings is related to our psychological association. Colors are very powerful. They have a strong appeal and temptation. Pink will make people feel sweet and vivid, but if a normal stays in a room which is decorated with too many pink elements for a long time, he will easily suffer from neurasthenia, or even being restless, which means it would be easy for him to have conflicts with others. Therefore, generally in interior space, especially in bedroom, the interior designers will not recommend pink paint.

On the contrary, the blue can bring a quiet and natural feeling, causing people's blood pressure stable, pulse beating soft and it can also effectively ease the mood swings, at the same time making people feel romantic and free. Blue is in line with people's visual psychological habits, not only giving a peaceful feeling but letting the brain relax as well. Using blue in the bedroom can make the room more intimate. And you may get more sleep at nights living in a blue atmosphere.

Green is a very natural color. According to an investigation, people in the green space will have their body temperature reduced by one to two degrees, at the same time, it can make people's breath and heart beat frequency slow down. In addition, green can also speed up the absorption of viscera digestion to achieve a good calming effect. Therefore, green is very helpful for ADHD patients to cool down their psychological pressure. All in all, it is a good idea to set green as the environmental color in interior space.

Yellow feels simple and neat, giving people a sense of vitality. In an indoor environment, set the bright and pure yellow can enhance people's logical thinking; promote people to develop good habits.

A variety of colors are easy to speed up body's blood circulation, making people's heartbeat faster, and improve people's ability to adapt. So in children's bedroom, the color of the application is generally more abundant than in an adult's room. While a variety of colors can also improve children's awareness sensitivity of color. However, if the interior space is filled up with too many different colors, it can make people irritable, so it's not a good idea to have too much color in one interior space.

\section{B. Color Character}

Studies have found that color not only can affect people's psychological and physiological reactions, but it is also a representation for the character. For example, pink represents a sweet, gentle and innocent character, generally the character of young women. Their emotional are fragile, very sensitive, and easy to produce the feeling of injury. In addition, the color also has a great impact on people's character. With the high-speed social operation, people's pace of life is speeding up rapidly. So their psychological pressure is also growing. They may often feel at a loss, depressed or restless. For this universal social psychology and behavior, the community is actively exploring effective ways of decompression. For interior design, color cannot be ignored on meditate people's mentality. Also, the role of color on the regulation of all kinds of character cannot be underestimated as well.

First of all, the color can be divided into cool and warm color. Representatives of The cold color are blue, green and purple; it can show the quiet and gentle side of a person. Representatives of the warm color are red, orange, yellow; it can show the friendly, warm side of a person. Therefore, in modern interior design, more and more people tend to create a complementary character for their own living space according to their own characters.

For example, people with blue character are calm and elegant. They are extremely dedicated and thoughtful.

However, people with a blue character are naturally suspicious, because of their particular attention to details, they are often hesitant and they procrastinate. They are also picky and distant. Therefore, in order to make up for the defects of the blue character, we can set the main color of an interior space blue and add a little warm color elements such as a red chair, an orange coffee table and so forth. This can give hurry to the blue character, so as to inspire their emotions, and stimulate them to make faster decisions or to complete a task.

While people with green character are optimistic about themselves, they are calm, easy to be close with others; they stand aloof from worldly success, pursuit a fairy natural state of life. But in daily life, they can be weak, indecisive. Therefore, in interior design, a space with natural, comfortable and harmonious background, we can add a little orange and other bright color. Because people with yellow character are quick, direct and bold. This will not only bring the whole living space some new and unique feelings, but also can make up for the indecisiveness of the green character.

People with yellow character have always been more logical, organized. Therefore, simple and straightforward design can meet their needs. We can match it with some mature complementary colors, such as a purple screen or carpet, etc. when matching colors; try not to pick colors with great impact on eyes. The appropriate choice would be choosing low tune colors, to ease stubborn and irritability of a yellow character, and also remember to reduce the brightness of the color in order to have a feeling of gentle and harmony.

\section{THE COLOR PRINCIPLE FOR INTERIOR DESIGN}

\section{A. Color Unity}

For Interior design, the principle of the unity of colors is based on a balanced mix of different colors. It requires similar brightness and purity in similar colors to show the same color style. If we combine many colors randomly, it would be unexpectedly unnatural in an interior environment. Unity principles need to utilize the generality of colors and deploy them properly, which can reduce the sense of visual confusion. 


\section{B. The Priority of Choosing Colors}

In interior design, because the furniture are often complex and big, the color of each object is also different, how to unify these colors into one same space environment without any sense of chaos is crucial, in general the principle is, if the overall indoor color looks dull, and dark, you can do some color on an appropriate area to enhance the vitality of the entire space. But it needs to be noted that the area of the decorative color should be strictly controlled. If the dotted area is too small, it may be assimilated by the surrounding color so that it cannot achieve the effect of embellishment, if the dotted area is too large, it would produce overwhelming and negative effect.

\section{Color Rhythm}

Color rhythm is quite similar to music rhythm. It means to arrange colors in their hue, brightness, purity, area, location and other contrast methods. The main techniques are repeat, gradient and so on. Repeat refers to the technique of repeating one color combination regularly, which can be very organized and regular. The rhythm of gradient refers to the technique of gradually changing the color hue, brightness, purity, location, area and other factors, so as to make the picture expressive and orderly. While Mutation is an art technique of bringing non-order factors into orders. This technique can break the tedium of a picture and increase the ups and downs of the rhythm. Movement is another technique. It can obtain a sense of movement rhythm through the use of bright or hue contrast to move the visual center of a picture to one side.

\section{Advancing With the Times}

The match of colors needs to undoubtedly advancing with the times. Although fashion is easy to die, but just because of it, we can constantly introduce new, endlessly giving people the sensory stimulation. The principle of the color with the times requires designers to focus on the everchanging fashion flow, and accurately grasp the popular colors of the current era. Through their own strategizing, they need to utilize those skills to interior design. This can bring dynamic changes to the daily life of people, so as to slow down people's aesthetic fatigue and enhance people's color perception.

\section{CONCLUSION}

As Arthor, a master from Finland, once said, when you design a window, imagine that your girlfriend is sitting right beside it. That is to say, an interior designer must dedicate his real feelings to every design and arrangement. With humanistic feelings in their hearts, interior designers should make the best place for human to live in. by utilizing color in interior design to create a great space atmosphere is a important trend for modern design. When we stay in an interior environment, not only do we want a visual feast, but a fulfillment in heart. A good interior environment can cultivate people's taste and keep our body and mind healthy because human can always feel the environment directly and resonate with everything surrounding us.

\section{REFERENCES}

[1] He Qingyan.Chinese Traditional Color and its Application in New Chinese Style Rooms.[J].Journal of Southeast University.2006.

[2] Hu Jianzhong The Art Features of Decorative Materials and its New Application in Interior Design.[J].Sichuan Institute of building research.2013.

[3] Liang Bing Emotional Design.[J].Art Observation.2008.

[4] Xiong YingJun, Hu Jinjun The Modern Hospital Environment Color Design.[J]. China's Journal of Hospital Administration.2005. 